\title{
El videojuego como dispositivo de (des)empoderamiento: La noción de agencia en el liberalismo avanzado / Video Games as (dis)Empowerment
}

\section{Devices: Agency in Advanced Liberalism}

\author{
*Daniel Muriel \\ Instituto de Estudios de Ocio. Universidad de Deusto. España/Spain \\ daniel.muriel@deusto.es \\ Recibido / Received: 22/12/2016 \\ Aceptado / Accepted: 26/10/2017
}

\section{RESUMEN}

Este artículo busca abordar la noción de agencia dentro del contexto del liberalismo avanzado a través del estudio de los videojuegos. El texto se apoya en los resultados de una investigación empírica más amplia que toma como premisa fundamental la existencia de una creciente cultura del videojuego en las sociedades contemporáneas. Desde un punto de vista teórico que bebe de los estudios sobre gubernamentalidad neo-foucaultianos, la teoría del actor-red, y los game studies, y utilizando una metodología cualitativa, el artículo considera que los videojuegos ayudan a visualizar, al mismo tiempo que fomentan, las transformaciones que en la noción de agencia operan a nivel ontológico, político y social: por un lado, un giro hacia el post-humanismo, las articulaciones entre elementos diferentes, la relacionalidad mediada y prostética; por otro, se observa la reproducción de las racionalidades neoliberales dominantes mientras se intuye, al mismo tiempo, su apertura hacia nuevas formas actanciales.

Palabras clave: Agencia, videojuegos, libertad, teoría del actor-red, liberalismo avanzado.

\section{ABSTRACT}

The aim of this article is to approach the notion of agency within Advanced Liberalism through the study of video games. The text is grounded in the findings of a research project that stems from a fundamental premise about the existence of a growing video game culture in contemporary society. From a theoretical point of view that builds on neo-Foucauldian governmentality studies, actor-network theory, and game studies, and using a qualitative methodology, the paper posits that video games help visualize, and also promote, the transformations that takes place in the notion of agency at a social, political, and ontological level: on the one hand, a shift to post-humanism, the articulation of different elements, and a mediated and prosthetic relationality; on the other hand, it is possible to observe the reproduction of the hegemonic neoliberal political rationalities while, at the same time, envisaging their aperture toward new agency forms.

Keywords: Agency, video games, freedom, actornetwork theory, advanced liberalism.

\footnotetext{
*Autor para correspondencia / Corresponding author: Daniel Muriel. Instituto de Estudios de Ocio. Universidad de Deusto. Avd. de las Universidades, 24. 48007 Bilbao, Bizkaia

Sugerencia de cita / Suggested citation: Muriel, D. (2018). El videojuego como dispositivo de (des)empoderamiento: La noción de agencia en el liberalismo avanzado. Revista Española de Sociología, 27 (3), 451-467

(Doi: http://dx.doi.org/10.22325/fes/res.2018.19)
} 


\section{INTRODUCCIÓN}

La interactividad es un sustantivo normalmente asociado a los videojuegos. De hecho, la naturaleza interactiva de los mismos es uno de los principales argumentos esgrimidos para diferenciarlos de otros productos culturales 0 medios: existe un pensamiento generalizado por el que los jugadores de videojuegos "no solamente consumen paquetes mediáticos preestablecidos, sino que, en cambio, son participantes activos en la creación de su experiencia" (Calleja, 2011: 56). Esto se ha señalado como la evidencia definitiva por la que los videojuegos deberían ser considerados la forma más pura de un nuevo paradigma de consumo cultural (Kirby, 2009: 167), uno que requiere de la participación explícita del jugador. Este planteamiento se contrapone frecuentemente a un modelo más vertical y pasivo, vinculado tradicionalmente a medios como la televisión, el cine o la literatura, dibujando un movimiento desde modelos sociales principalmente asociados al "espectáculo" (Debord, 1995) a otros centrados en la "participación" (Jenkins, 2006).

En este sentido, los videojuegos siempre han sido celebrados por ofrecer opciones para aquellos que deciden jugarlos, pero este atributo se ha tornado especialmente reflexivo en los últimos años. En este sentido, han emergido un gran número de títulos que se aproximan explícitamente a la idea de agencia en el jugador. Por ejemplo, Until Dawn (Supermassive Games, 2015) y Life is Strange (Dontnod Entertainment, 2015) utilizan las metáforas e imaginario del efecto mariposa para trasladar al jugador la idea de que todo lo que hace, no importa cuán pequeña sea la acción, afectará a la trama y al destino de los personajes. Similarmente, Dreamfall Chapters (Red Thread Games, 2014) avisa a sus jugadores de que "el balance ha cambiado" cada vez que toman una decisión importante. Los trabajos recientes de TellTale Games tales como The Walking Dead (temporada 1, 2012; temporada 2, 2013; temporada 3, 2017), The Wolf Among Us (2013), Game of Thrones (2014), Tales from the Borderlands (2014), o Batman: The TellTale series, le indican al jugador que la historia será confeccionada de acuerdo a sus decisiones. Otros títulos como Heavy Rain (2010), Beyond: Two Souls (2013) y Detroit (2018) de Quantic Dream, juegan con las mismas ideas y, en su caso, ofrecen múltiples finales dependiendo de las decisiones tomadas. Muchos de estos juegos, además, muestran al final de cada capítulo datos sobre las elecciones realizadas, permitiendo que cada jugador compare sus propias acciones con las del resto de la población que ha jugado esa obra. La cuestión de la capacidad del jugador de realizar cambios significativos se encuentra en el centro de esta tendencia dentro de los videojuegos y esto hace que ocupen, por lo tanto, una posición privilegiada para entender la agencia en la actualidad.

Apoyándome en una investigación empírica de corte cualitativo más amplia sobre la cultura del videojuego, este artículo se propone entender algunas de las aristas de la noción de agencia en un contexto de modernidad avanzada. Se trata de observar cómo actores relevantes dentro de la cultura del videojuego problematizan continuamente la agencia, abriendo la oportunidad de examinar esta importante noción tanto en relación con las prácticas asociadas al videojuego como aquellas que hacen referencia a un espectro social más amplio.

Así, en primer lugar, y tomando como referencia la teoría del actor-red (Latour, 2007; Law, 2004), definiré la agencia como la producción múltiple, distribuida y dislocada de diferencias y transformaciones que puede tomar multitud de formas. Considero que es crucial extender la definición de agencia más allá de su límite tradicional, el del ser humano. Los videojuegos y los dispositivos en los que son producidos deberían ser incluidos también en su análisis (Giddings, 2009).

Después, discutiré la idea de libertad como condición de posibilidad de los jugadores para expresar su agencia. Los videojuegos se presentan llenos de oportunidades y decisiones que tomar, y la responsabilidad de actuar es depositada en el jugador: ¿Qué camino tomar? ¿Quién debería sobrevivir? ¿Qué acción llevar a cabo? La libertad es entendida aquí en términos neo-foucaultianos, como parte de las racionalidades políticas neoliberales en las que los ciudadanos son gobernados a través de su libertad para elegir (Miller and Rose, 2008: 82). A partir de aquí, dos tendencias emergen. Por un lado, hacia un conjunto de prácticas 
y discursos de empoderamiento: estar en control de la situación, superar el reto, la lógica del logro (Consalvo, 2007). Por otro lado, la atención se centra en los procesos de desempoderamiento: fracaso (Juul, 2013), frustración, pérdida de agencia.

A continuación, esta particular construcción de la agencia es trasladada a la vida cotidiana, con resultados dispares. Por una parte, los videojuegos pueden aumentar el alcance social de la agencia, articulando un tipo de agencia extendi$\mathrm{da}^{1}$ que, en algunas ocasiones, posibilita transferir destrezas desde experiencias de juego particulares a escenarios de la vida cotidiana. Por el contrario, los videojuegos también son capaces de reducir el alcance social de la agencia al absorber la atención de los jugadores y su capacidad para interactuar socialmente.

Finalmente, dibujo en las conclusiones lo que los videojuegos nos dicen acerca de la agencia en la contemporaneidad a nivel ontológico, político y social. En ellas se vislumbran las promesas (y amenazas) de la agencia dentro de los dispositivos del neoliberalismo y más allá. El videojuego se constituye, probablemente, como uno de los escenarios actuales donde mejor se pueden observar las disputas ontopolíticas sobre la agencia.

\section{METODOLOGÍA}

Este texto se apoya parcialmente en un proyecto de investigación más amplio sobre la cultura del videojuego. En él, no sólo se plantea lo que la sociología puede decir del videojuego como experiencia, cultura, o entramado sociotécnico, sino que también busca entender cómo los videojuegos y su cultura nos pueden ayudar a entender cuestiones como la agencia, el poder, lo cotidiano, las identidades o los procesos de subjetivación en las sociedades contemporáneas. Se trata de abordar cuestiones de hondo calado social en la contemporaneidad a través del videojuego, es decir, como realidad social

1 Se entiende por agencia extendida aquella agencia que se apoya en otros medios para transformar ciertos aspectos de la realidad a distancia (temporal y espacialmente). en la que se reflejan (y fomentan) muchos de esos procesos, entre ellos, cuestiones relacionadas con la identidad, la experiencia, la empatía, la cultura, 0 las racionalidades políticas hegemónicas. En este caso se toman los resultados que giran en torno a la cuestión de la agencia.

En el conjunto de la investigación se realizaron 28 entrevistas semi-estructuradas (en este texto se citan 16 entrevistados, es decir, corresponden a más de la mitad de las entrevistas realizadas), principalmente a residentes en Reino Unido y España, aunque también hubo entrevistados de otros países europeos (Alemania, Suecia, Luxemburgo) y de Estados Unidos - hubo entrevistas cara a cara, pero también vía skype (en una proporción del cincuenta por ciento)-. Los entrevistados fueron seleccionados siguiendo el criterio de incluir un amplio rango de roles y grupos dentro del mundo del videojuego (las categorías no son necesariamente excluyentes): jugadores, desarrolladores (diseñadores, programadores, artistas), académicos, periodistas de videojuegos, youtubers, directores de museos y centros de interpretación sobre videojuegos. Mientras la mayoría de estudios empíricos sobre la cultura del videojuego se centran en un tipo particular de actor, esta investigación explora la diversidad de esta cultura y sus redes desde múltiples perspectivas. El motivo de utilizar un abanico tan heterogéneo de personas radica en el hecho de que la investigación buscaba aprehender fenómenos, aunque relacionados, diversos, con la intención, por lo tanto, de obtener el mayor número de enfoques posibles dentro de los estándares de un diseño empírico de corte cualitativo. En cualquier caso, los fragmentos de entrevistas utilizadas en este texto son representativas del conjunto de discursos que giraron alrededor de la cuestión de la agencia. Así, aunque hayan quedado ciertas entrevistas por citar, lo que aquí se muestra expresa de forma representativa la totalidad del trabajo empírico realizado. Todos los participantes citados en este texto lo hacen bajo seudónimo.

En relación con la categoría de jugadores de videojuegos, los entrevistados fueron elegidos de acuerdo a cómo ellos percibían su nivel de compromiso con la cultura del videojuego y a su propia 
identificación —o no- como videojugadores 0 gamers. Los casos se sitúan en una escala continua que va de aquellos que tienen una alta implicación en la cultura y se consideran gamers y aquellos menos activos y que no se identifican necesariamente como tal. Las entrevistas con miembros de la industria del videojuego fueron escogidas de acuerdo al rol que desempeñaban (productores, managers, programadores, diseñadores, artistas, marketing, atención al usuario), al tipo de compañía (tamaño, si eran desarrolladores y/o editores), y el tipo de videojuegos con los que trabajaban (AAA, indie, juegos free-to-play). Los entrevistados categorizados como pertenecientes a los medios de comunicación, fueron seleccionados en relación con el tipo primario de medio (escrito 0 audiovisual) y respecto al rol desempeñado (director, redactor, creador de contenidos). Finalmente, también fueron entrevistados individuos que trabajaban en el sector de la cultura y el arte en relación con los videojuegos, entre ellos, directores de museos y otros centros de exhibición e interpretación del videojuego.

En todos los casos se tuvo en cuenta el género y la edad de los participantes, con la intención de obtener un rango lo más diverso posible. El rango de edad se movió entre los 24 y 54 años, mientras que la estructura de género quedó en 7 mujeres y 21 hombres (en la categoría de jugadores la distribución es 50-50, pero en el resto se refleja la casuística de un espacio dominado por el género masculino). Adicionalmente, de todos los entrevistados se tomaron notas de conversaciones y comunicaciones más informales para alimentar la investigación, incluyendo información extra sobre su trabajo y experiencias vitales.

Más allá de las entrevistas, y teniendo en cuenta que los videojuegos pueden ser considerados también como "participantes" activos en esta investigación, se ha utilizado la acción de jugar como una técnica de investigación más (Mäyrä, 2008: 165-167; Karppi and Sotamaa, 2012). En este sentido, además de jugar de un modo más analítico, intenté involucrarme de forma más profunda con la cultura del videojuego. Esto supuso principalmente una observación mucho más regular e intensa de la cultura online y offline del videojuego, incluyendo un acercamiento a revistas, blogs, críticas, foros, y canales de Youtube y Twitch ${ }^{2}$ sobre videojuegos. Por ejemplo, revistas, sitios web y blogs como Rock, Paper, Shotgun ${ }^{3}$, Polygon $^{4}, K_{\text {Kotaku }}^{5}$, GiantBomb $b^{6}$, Gamasutra $^{7}$, , Waypoint ${ }^{8}$, PC Gamer ${ }^{9}$, Eurogamer ${ }^{10}$, Kill Screen ${ }^{11}$, AnaitGames ${ }^{12}$, Zehngames ${ }^{13}$, Deus Ex Machina ${ }^{14}$, AntiHype ${ }^{15}$, o Presura ${ }^{16}$. En relación con Youtube y Twitch, seguí de forma regular canals como los de PewDiePie ${ }^{17}$, Feminist Frequency ${ }^{18}$, TotalBiscuit ${ }^{19}$, The Syndicate Project ${ }^{20}$, Pushing Up Roses ${ }^{21}$, PBS Game Show'22, Jim Sterling23, Scanliner $^{24}$, DayoScript ${ }^{25}$, Bukku qui ${ }^{26}$, SonyaTheEvir27, Fremily $^{28}$, y Littlemisspiss, entre otros. También hice un seguimiento de comentarios realizados en redes sociales como Twitter, Facebook o Reddit.

De forma adicional, se realizaron una serie de observaciones online siguiendo determinados videojuegos (Gone Home, This War of Mine, Titan

2 Twitch es una plataforma online en la que es posible emitir en directo partidas de videojuegos, permitiendo que cualquiera pueda unirse a su visionado y en la que se habilita un chat en tiempo real para quienes quieran comentar lo que allí está sucediendo (https:// go.twitch.tv/). https://www.rockpapershotgun.com/

4 https://www.polygon.com/

5 http://kotaku.com/

6 https://www.giantbomb.com/

7 http://www.gamasutra.com/

8 https://waypoint.vice.com/en_us

9 http://www.pcgamer.com/

10 http://www.eurogamer.net/

11 https://killscreen.com/

12 http://www.anaitgames.com/

13 http://www.zehngames.com/

14 http://deusexmachina.es/

15 http://antihype.es/

16 http://www.presura.es/

17 https://www.youtube.com/user/PewDiePie

18 https://www.youtube.com/user/feministfrequency

19 https://www.youtube.com/user/TotalHalibut

$20 \mathrm{https}: / /$ www.youtube.com/user/TheSyndicateProject

21 https://www.youtube.com/user/pushinguproses

22 https://www.youtube.com/user/pbsgameshow

23 https://www.youtube.com/user/JimSterling

24 https://www.youtube.com/channel/UCWz51s7gdp55UXY_xm69FQ

25 https://www.youtube.com/user/DayoScript

26 https://www.youtube.com/channel/UCG01J/3yFtHJbTnoZgeoKZg

27 https://www.twitch.tv/sonyatheevil

28 https://www.twitch.tv/fremily 
Souls, Papers, Please, Gods Will Be Watching, Watch Dogs, Life is Strange, Skyrim y Bloodborne). Estas observaciones se basaron en seguir las experiencias que se asociaban a esos videojuegos transmitidas por tres actores principales: comunidades de jugadores en foros, redes sociales y en comentarios de reseñas; profesionales del medio en páginas web especializadas; intervenciones de desarrolladores en medios de comunicación y en conversación directa con ellos. Todos estos juegos -y algunos más - fueron al mismo tiempo jugados por el autor de este texto, registrando y usando sus propias experiencias como parte del desarrollo metodológico. En un contexto social atravesado por una importante cultura digital, este tipo de aproximación es crucial para entender las realidades sociales que se forman alrededor de ella. Entre otros elementos, Twitter, Facebook, Reddit, Instagram, YouTube, sitios web, blogs, y los propios videojuegos conforman un espacio social donde se dan forma a las relaciones sociales.

\section{AGENCIA Y VIDEOJUEGOS DESDE UNA SOCIOLOGÍA DE LAS MEDIACIONES}

Antes de explorar qué nos puede decir la cultura de los videojuegos sobre la agencia en las sociedades contemporáneas, plantearé una definición de agencia que me permita desbrozar sus características principales: la agencia es la producción múltiple, distribuida y dislocada de diferencias y transformaciones que puede tomar multitud de caracterizaciones en forma de un agente, actor 0 personaje determinado (Muriel, 2016).

La primera característica de la agencia es que existe en tanto que transforma de algún modo la realidad (Muriel, 2016: 115-116). La agencia por lo tanto no tiene tanto que ver con la intencionalidad, deseo 0 volición de un actor —y su mayor o menor racionalidad - como con las transformaciones que opera, que son las diferencias efectivamente observables y rastreables:

Sin una explicación, sin pruebas, sin diferencias, sin transformación de algún estado de cosas, no hay razonamiento significativo a hacer respecto de una agencia, ningún marco de referencia detec- table. Una agencia invisible que no produce ninguna diferencia, ninguna transformación, no deja rastro y no aparece en ningún relato no es una agencia. Punto. Hace algo 0 no (Latour, 2008: 82).

He ahí el núcleo de la fundamentación de toda agencia: 0 produce algún tipo de cambio 0 es que no existe. La agencia de un actor determinado, entonces, sólo puede ser definida "a través de la observación de sus actos” (Latour, 2001: 147). Esta es la razón por la que los videojuegos — pero también el hardware, las conexiones y los periféricos que hacen posible la interacción-, y no sólo los jugadores, pueden ser considerados como actores. Ambos actúan uno sobre el otro, transformando sus respectivos cursos de acción: "El jugador, antes que actuar, reacciona a lo que el videojuego pone delante de él y, de forma similar, el juego reacciona a esta interacción" (Arsenault and Perron, 2009: 119-120). En este caso, la "distinción entre las capacidades de agencia de humanos y no-humanos deja de ser útil" (Sayes 2014: 145). El lenguaje de las personas entrevistadas refleja la agencia de los videojuegos al reconocer cómo tienen un impacto sobre la realidad, en particular sobre aquellos que los juegan. Así, es posible observar que los videojuegos 'pueden hacer que la gente se reúna' (Andrew, hombre, 28, muy involucrado en la cultura del videojuego y se identifica como gamer), "proveer de un sentido de amistad', (Zelda, mujer, 25, muy involucrada en la cultura del videojuego y se identifica parcialmente como gamer), 'tienen un efecto en los jugadores' (Steffan, hombre, 45, manager en una universidad de videojuegos), 0 incluso 'sobrepasarte y hacerte pensar las cosas dos veces' (Laura, mujer, 26, desarrolladora de videojuegos independientes).

La segunda característica de la agencia es que es múltiple y no descansa en ningún actor prototípico (Muriel, 2016: 116). Si bien la agencia es la acción genérica que provoca cambios en el mundo, siempre tiene una figuración (Latour, 2008: 83), se encarna en algún tipo de forma, figura o personaje. Pueden encarnarse en fórmulas muy distintas sin pasar por ningún actor estándar 0 que deba considerarse social en el sentido que tradicionalmente se le ha podido dar en la sociología (ibídem: 85-86). Con describir y registrar aquellas figuraciones que 
encontremos, sin necesidad de filtrarlas 0 disciplinarlas a priori (ibídem: 86), ya comenzaríamos a observar la multiplicidad de la agencia.

Así, es posible "resaltar el carácter abierto de la agencia, que puede ser ocupada por las más heterogéneas mezclas de humanos y no-humanos" (García Selgas, 2007: 144). La libertad para definir al actor es enorme: desde entenderlo como una entidad tremendamente compleja y abstracta (las instituciones, el colectivo de gais y lesbianas, la red ferroviaria, el cuerpo humano, la estructura social) hasta pensarlo como un personaje concreto (el presidente del gobierno, Dios, el autor de este texto, un videojuego específico). En definitiva, se trata de reconocer el heteromorfismo de la agencia.

La agencia también es múltiple en tanto que se configura relacionalmente. Para que alguien 0 algo (una institución, una persona, un virus) se sitúe como una personificación y actúe como agente social, se tiene que dar una interacción entre factores de diversa naturaleza (biológicos, materiales, tecnológicos, semióticos), al mismo tiempo que se necesita de "la relación contingente y siempre cargada de desigual poder con los otros", es decir, de las otras agencias, que determinen como consecuencia que "su relacionalidad es constitutiva y funcional” (García Selgas, 2007: 140). Que después aparezcan en posiciones-sujeto o personificaciones concretas - envueltas, institucionalizadas, estabilizadas - no deja de ser un efecto, un resultado. Los videojuegos, las plataformas para jugar y sus redes, los jugadores, los desarrolladores, los sitios web y canales de emisión de videojuegos, y las representaciones artísticas y socioculturales del videojuego son, al final, el resultado de asociaciones complejas entre diferentes actores humanos y no-humanos.

La última característica de la agencia la describe como distribuida y dislocada (Muriel, 2016: 117). Esta noción de agencia ignora "la alternativa entre actor y sistema" (ibídem: 306), gestionando el dualismo acción/estructura que arrastra la disciplina desde sus comienzos. Entendiendo que la acción no nace en la conciencia, el hecho de que el individuo casi nunca puede hacer lo que quiere hacer no se explicaría atribuyéndolo a una fuerza social externa como el habitus, la sociedad, el gru- po 0 cualquier otra realidad determinante que se apropiara de la conducta (Latour, 2008: 69-73). La explicación descansaría en la idea de que la acción se encuentra dislocada (ibídem: 74-75), no reducible a categorías sociales predefinidas como las descritas. La acción, por lo tanto, no es el producto directo del actor, pero tampoco lo sería de la estructura en la que, en términos convencionales, se insertaría. Latour relata el origen de su planteamiento como necesidad de dar cuenta de dos insatisfacciones que persiguen a todos los sociólogos:

- Primero, enfrentados a lo que se suele llamar el nivel micro (la interacción cara a cara, lo local), los científicos sociales se percatan que muchas de las cosas que necesitan para dar sentido a la situación vienen de muy lejos en el tiempo y el espacio. Esto les fuerza a buscar en otro nivel (la sociedad, las normas, los valores, la cultura, el contexto, la estructura), concentrándose "en lo que no es directamente visible en la situación pero que ha hecho posible la situación tal como es" (Latour, 1999: 17).

- Segundo, una vez que es alcanzado ese nivel superior, el nivel macro, los científicos sociales sienten que les falta algo, ya que abstracciones como las de cultura, sociedad, valores 0 estructura parecen demasiado grandes, por lo que vuelven a buscar las situaciones locales, las de carne y hueso, de las que habían partido previamente.

Lo que nos permite entonces un enfoque sociológico centrado en las mediaciones es poder atender a estas insatisfacciones sin intentar superarlas o resolverlas. Lo social no estaría hecho "en absoluto ni de agencia, ni de estructura, sino más bien de ser una entidad circulante" (ibídem). № existiría, por lo tanto, ni la estructura ni la acción como lugares específicos o sustancias, sólo movimientos, conexiones, asociaciones y mediaciones que, ocasionalmente, pueden dar lugar a entidades concretas y contingentes. La naturaleza distribuida de la agencia es algo que se ve claramente en el ámbito de los videojuegos:

Debemos resistirnos a entender el videojuego como un objeto "total" y discreto. El videojuego está constituido por un software que efectúa sus propias operaciones y posee una agencia semiautónoma 
dentro del sistema de juego. Los mundos y temporalidades del juego, modos de presentación, puzles y combates, la identificación con los personajes controlados por el sistema, están continuamente configurando la experiencia del jugador y reaccionando a sus intervenciones (Giddings, 2009: 148).

\section{LIBERTAD, RESPONSABILIDAD Y PROCESOS DE (DES)EMPODERAMIENTO EN LOS VIDEOJUEGOS: LA CULTURA DEL VIDEOJUEGO EN EL LIBERALISMO AVANZADO}

Libertad es una noción clave tanto en el universo de los videojuegos como en el neoliberalismo. Es parte de un vocabulario que articula los discursos y experiencias en torno a ambos.

En el liberalismo avanzado, fruto de un progresivo adelgazamiento del Estado, las relaciones entre lo público y lo privado son resignificadas en lo que Gordon (1991: 36) denomina modos de pluralización del gobierno moderno: lo social y la cuestión gubernamental ya no descansan exclusivamente en el Ilamado Estado-providencia (Donzelot, 2007: 176), sino que se apoya en una red de agentes que lo atraviesan, lo transcienden o se sitúan en su periferia como organismos sub y supra estatales, ONG, consultorías, think tanks, conglomerados de medios de comunicación, lobbies, partidos políticos, organizaciones sociales y comunitarias de diverso tipo. Es una economización de los medios de gobierno estatales en la que se sirve y aprovecha de la propia energía de los gobernados. En palabras de De Marinis, el neoliberalismo busca "gobernar contando con la mayor cantidad posible de la energía que para su propio gobierno aporten los gobernados mismos" (2005: 22). Se perfila una sociedad de sujetos responsables de su propio gobierno, bienestar e identidad: los poderes del Estado van dirigidos por lo tanto al empoderamiento de sujetos emprendedores con capacidad de elección. Se trata de "la conversión de la exigencia de soberanía en mandato de autonomía" (Donzelot, 2007: 177), por la que el Estado adelgaza al tiempo que se genera un individuo como "empresario de sí mismo" (Gordon, 1991: 44). Esta nueva realidad post keynesiana requiere de un sujeto que se autorregule, que "sea activo, responsable, participativo, dinámico" (De Marinis, 2005: 25). Es la lógica fundamental del neoliberalismo por la que "los individuos pueden ser gobernados a través de su libertad para elegir" (Miller y Rose, 2008: 82). Por lo tanto, libertad y control, autonomía individual y gobierno, son pares que no se encuentran reñidos, forman parte indisociable de una nueva fórmula en el manejo y definición de realidades, donde la idea de libertad es fundamental.

Así, las racionalidades políticas predominantes en nuestra sociedad son las del liberalismo avanzado 0 neoliberalismo. Aquí, antes que entenderlo como un modelo económico, estas racionalidades que atraviesan lo social contemporáneo son una forma hegemónica de conducir la conducta de individuos y poblaciones. Esto es, enfrentamos, principalmente, un problema de primer orden sociológico y que está relacionado con las posibilidades y limitaciones de la agencia. Por ello, las racionalidades políticas pueden entenderse, según Miller y Rose (2008), como las regularidades en el discurso político en la "formulación y justificación de esquemas idealizados para la representación, análisis y rectificación de la realidad" (2008: 58). Tres son sus características principales (ibídem: 58-59).

Primero, poseen una típica forma moral: se basan en principios que deberían guiar las tareas de gobierno (libertad, justicia, igualdad, mutua responsabilidad, ciudadanía, sentido común, eficiencia económica, prosperidad, crecimiento, imparcialidad, racionalidad). Segundo, tienen un carácter epistemológico: son articuladas en función de una idea de la naturaleza de los objetos - la sociedad, la nación, la población, la economía- a gobernar. Tercero, se constituyen como un lenguaje específico: un tipo de lenguaje que funciona como un aparato intelectual para hacer pensable la realidad, lista para entrar dentro de las deliberaciones políticas. En definitiva, y resumiendo estas tres características, las racionalidades políticas se encuentran "moralmente impregnadas, cimentadas en el conocimiento y hechas pensables a través del lenguaje" (ibídem: 59).

En este sentido, los videojuegos también reproducen un esquema de libertad como piedra de toque que sostiene su estructura. Los jugadores sienten a 
menudo que pueden actuar sin restricciones dentro del universo de juego, llevándoles incluso a ignorar algunas de las tareas que el videojuego les manda hacer. Así es como lo expresa uno de los entrevistados, que trabaja en el ámbito del cine documental produciendo contenidos relacionados con el videojuego, refiriéndose a Shadow of the Colossus (Team Ic0, 2005):

Shadow of the Colossus. Es un mundo enorme del cual solo necesitas atravesar ciertos puntos para ir de un punto $\mathrm{A}$ a un punto $\mathrm{B}$. Eso sí, la libertad de tu dedicarte a pasear con el caballo, por todo ese sitio por zonas a las que no necesitas ir (...), eso está ahí. Y dices, yo tengo la libertad de pasearme por esa tierra, ¿sabes? (Isidoro, hombre, 27, director de documental sobre videojuegos).

Es la idea que está detrás de lo que en el ámbito de los videojuegos se conoce como "mundos abiertos", que no sólo atañe a juegos online masivos persistentes como World of Warcraft (Blizzard Entertainment, 2004) o Eve Online (CCP Games, 2003), sino que también incluye obras —ligadas al género sandbox ${ }^{29}$ - que consisten en extensos mapeados que permiten un juego no lineal, facilitando narrativas emergentes. Estos títulos se basan en dar libertad al jugador para explorar el universo de juego y actuar sin seguir un guión preestablecido de antemano (dentro de las limitaciones que ofrece el sistema), permitiéndole elegir las misiones que desea llevar a cabo, en qué orden, e inundando la historia de tareas secundarias $0 \mathrm{mi}-$ nijuegos (que son una suerte de juegos dentro del juego). Las sagas Elder Scrolls, The Witcher, Grand Theft Auto, Assassin's Creed, Fallout y Mass Effect son algunos de los ejemplos paradigmáticos de este enfoque que articula la experiencia de juego en torno a la idea de libre albedrío, que no es más que la maximización del principio que parece regir todo videojuego de acuerdo a su retórica: la libertad de acción del jugador.

Cada vez que decidimos ignorar las misiones de la trama principal y pasear libremente por

29 En el ámbito del videojuego, el sandbox es un género en el que el jugador está menos dirigido y constreñido por la estructura de juego, por lo que tiene libertad para moverse por su universo sin necesariamente tener unos objetivos muy definidos.
Liberty City o Tamriel (escenarios de las sagas Grand Theft Auto y The Elders Scrolls respectivamente), seleccionar la siguiente línea de diálogo que definirá nuestra relación con otros personajes, o seguir todos esos puntos que inundan los mapas con la siguiente tarea a realizar, estamos reproduciendo la idea de libertad que sostiene al liberalismo avanzado donde los individuos no son "meramente libres de elegir, sino que son obligados a elegir, a entender y vivir sus vidas en términos de elección" (Rose, 1999: 87). Siguiendo a Foucault, Nikolas Rose (1999) considera la noción de libertad, no como lo que se opone al poder, sino como su condición de posibilidad. No existe, pues, poder sin libertad — la posibilidad de elegir — del mismo modo que no existe libertad que no haya nacido en la tensión de complejas relaciones de poder. En palabras de Foucault, es necesario "un campo de posibilidades en los que distintos tipos de conducta, distintas formas de reaccionar y modos de comportamiento estén disponibles (2003: 139). Esta obligación se traslada al universo del videojuego en forma de una demanda por ejercer nuestra influencia y sentir la posibilidad de actuar en consecuencia:

Cuando uno mira a los videojuegos, se puede ver claramente, por ejemplo, nuestra demanda de sentir que tenemos capacidad de influencia, que tenemos la opción de interactuar (Bernard, hombre, 51, director de un museo sobre videojuegos).

La opción de interactuar, elegir, y decidir es para estos actores fundamental en los videojuegos. En última instancia, "los videojuegos van sobre tomar decisiones" (Patxi, hombre, 38, desarrollador, programador) y "elegir tu propio camino" (Minerva, mujer, 37, desarrolladora, artista). En este sentido, los videojuegos claramente buscan individuos activos, promocionando la producción de agentes participativos porque, de otro modo, la articulación de estos dos elementos (jugadores y videojuegos) no sería posible:

Es lo que convierte al videojuego en algo activo en oposición a algo pasivo. De hecho, tú tienes que ir y hacer algo para que algo ocurra 0, si no, nada ocurrirá (Albert, hombre, 25, desarrollador, artista).

Esto es paradigmático de cómo un sujeto activo es fundamental para entender la agencia cuando 
se trata de jugar a videojuegos, lo que sitúa al jugador en una posición central. Taylor compara a los jugadores con trabajadores sociales que "actúan como agentes productivos centrales en la cultura del videojuego" (2006: 159). Incluso va más allá y afirma que "no hay cultura, no hay juego, sin la participación del jugador" (Taylor, 2006: 159). Es el mismo punto de vista defendido por Egenfeldt-Nielsen et al., quienes consideran que los videojuegos demandan que los jugadores interactúen explícitamente con ellos, requiriendo al "usuario involucrarse con el juego" (2008: 138-139). Es más, la participación del jugador con los videojuegos parece ser esencial, "eres tú quién realmente gana y pierde" (Kirby, 2009: 169).

Esta es pues la condición de posibilidad de la agencia en el entorno de los videojuegos: libertad para elegir y la construcción de un sujeto activo dentro de ciertos parámetros. Por lo tanto, es importante considerar los videojuegos como dispositivos tanto facilitadores como condicionantes de la agencia: jugadores que experimentan sentimientos de dominio y poder tanto como de frustración y pérdida.

\section{El jugador como sujeto poderoso}

La mayoría de representaciones del jugador de videojuegos, por lo tanto, giran alrededor de la idea de que él es el protagonista, es quien está al cargo de manipular el flujo de lo que ocurre en la pantalla. Acabo de asegurar que la condición de posibilidad de la agencia dentro de los videojuegos requiere de libertad y sujetos activos, lo que conduce a la asunción común de que los videojuegos representan una forma cultural (más) interactiva:

Leer está genial, pero leer, tú siempre eres un observador en esa acción, mientras que, con los videojuegos, sin embargo, ya no eres un observador, tú estás realmente en esa historia, dentro de ella (Alfred, hombre, 26, fuerte identificación como jugador).

De acuerdo con este entrevistado, los jugadores no observan simplemente, ellos están en la historia, pertenecen y son una parte operativa de los procesos narrativos y mecánicos del juego. Los videojuegos fuerzan a los jugadores a participar activamente, a "pensar que tú eres el que está jugando, que lo que le pasa al personaje te está pasando a ti" (Patxi, hombre, 38, desarrollador de videojuegos, programador). Esto es un sentir generalizado entre la mayoría de entrevistados, lo que les lleva a reclamar que "tú sientes casi que eres el que está dentro de la pantalla" (Iker, hombre, 42, jugador ocasional). De nuevo, la imagen del sujeto neoliberal que es responsable de lo que (le) ocurre, se proyecta en estas afirmaciones. Los videojuegos fomentan esta visión de formas explícitas (como en esa serie de títulos citadas al principio, donde se le recuerda continuamente al jugador que sus decisiones influirán sustancialmente en el devenir de la historia contada) e implícitas (usando una perspectiva en primera persona, inundando la pantalla con misiones, submisiones y elementos a descubrir, 0 permitiendo fórmulas de personalización de los personajes y universo de juego).

Esta conceptualización de los jugadores los coloca en el centro de la discusión sobre la agencia, lo que facilita el reconocimiento de los jugadores como los actores sociales más importantes en la cultura del videojuego (algo que, como mostré en el apartado anterior, el planteamiento de este texto cuestiona). No es sorprendente por lo tanto que exista un discurso centrado en cómo los jugadores están al mando de la situación. Los videojuegos sólo pueden ser definidos como tales si tú, como jugador, "estás a los mandos" y, además, "controlas lo que está pasando" (Isidoro, hombre, 27, director de documental sobre videojuegos). En este contexto, los videojuegos tratan sobre "fantasías de poder" (Peter, hombre, 54, director de master sobre videojuegos):

La razón por la que juegos como Candy Crush son tan populares es porque te dan una sensación de control sobre tu vida cotidiana que normalmente no tienes. (...) Las cosas son como deberían ser en tu vida real. El trabajo duro es recompensado, tú tienes el control (Darius, hombre, 28, desarrollador, diseñador).

Este planteamiento retrata a los jugadores como sujetos poderosos, que tienen la capacidad de controlar el resultado de sus acciones de un modo que sólo podrían imaginar en sus vidas cotidianas. Los videojuegos crean un entorno donde 
la agencia de los jugadores se sobredimensiona, promocionando un sentido de poder y logro. De un modo más o menos explícito, los videojuegos normalmente cuentan a los jugadores que ellos tienen la habilidad de triunfar y superar retos:

Existe en ocasiones un mensaje sutil, pero a veces también muy explícito, que tiene que ver con el empoderamiento. Tú puedes hacerlo. Tú puedes hacer esto (Emmett, hombre, 47, director de un centro sobre videojuegos).

Los videojuegos producen las oportunidades para construir estas narrativas de éxito y triunfo, donde el jugador es el ganador y quien puede hacer lo que se proponga. Los sistemas de juego se sirven de diversas formas para llevar de la mano al jugador sin que lo aparente y otorgarle así esa envoltura de poder: puntos de vida que se regeneran automáticamente, sistemas de navegación y señalización de objetivos y enemigos (y aquí da igual si es una fantasía de corte medieval como The Witcher, una ambientación contemporánea como GTA, 0 un escenario futurista como Deus Ex) que, junto sistemas de pistas in-game, evitan que nos desorientemos o no sepamos qué hacer durante mucho tiempo, numerosos puntos de salvado, abundante munición, inteligencia artificial que se adapta a nuestra forma de jugar (es decir, se vuelve más fácil o difícil en función de nuestro rendimiento), y un largo etcétera.

Es una proyección de un individuo empoderado y con determinación que puede superar cualquier obstáculo en su camino. Esta representación de la agencia cae en la clásica identificación de los humanos como sujetos activos, intencionales y conscientes, en oposición a entidades no-humanas como objetos inconscientes, pasivos y sin intencionalidad. Es una forma de entender la agencia que no está alejada de las metanarrativas modernas que celebran "la emancipación del sujeto racional o trabajador" (Lyotard, 1984: xxIII), todavía fuertemente ancladas en el imaginario filosófico y político del (neo-)liberalismo. La promoción de individuos activos y participativos, entonces, no puede únicamente reducirse a una disposición en algunas formas subculturales, sino que está conectado a tendencias más amplias que afectan a la sociedad en su conjunto, y que, en este caso, coinciden con las racionalidades del liberalismo avanzado predominantes.

\section{Hacia la (no) paradójica narrativa de desempoderamiento y triunfo en la cultura del videojuego}

A pesar de la extendida proyección del jugador como un sujeto poderoso, después de jugar a This War of Mine (11 bit studios, 2014), es fácil percatarse de que existen juegos que buscan desempoderarnos de forma explícita. En esta obra, controlamos a un grupo de civiles que se encuentra en una ciudad sitiada en guerra. Vivimos en unas condiciones de escasez en cuanto a provisiones (comida, agua, medicinas) y abrigo (una casa en un estado de semirruina), lo que fuerza al jugador a buscar suministros y materiales en peligrosas escapadas nocturnas (cuando es más difícil que los francotiradores estén activos). Nos enfrentaremos a todo tipo de problemas y dilemas morales mientras intentamos proteger y ayudar a sobrevivir a nuestros personajes: alimentándolos, cuidando de su salud (mental y física), gestionando recursos para mantenerlos en calor, y defendiéndolos de agresiones externas. La falta de tutorial deja al jugador a su suerte; el juego le desorienta, puesto que no sabe cómo funcionan las cosas, qué hacer, cómo hacerlas. Como Pawel Miechowski, uno de sus desarrolladores, comenta: "cuando estalla la guerra nadie te dice qué hacer, no hay tutorial" (Warr, 2014).

De hecho, This War of Mine me hizo vivir una de las experiencias más devastadoras jugando a un videojuego. Llegó un momento en el que me percaté de que mis personajes no iban a sobrevivir, que todo estaba perdido. Sin embargo, el final no era inmediato, sabía que era inevitable, pero el sistema de juego seguía funcionando. Dos de mis personajes, debido a una depresión profunda causada por los acontecimientos vividos, estaban en shock, casi en estado catatónico. Era imposible hacer nada con ellos. Sólo podía controlar a un tercer personaje, que era, de facto, una especie de muerto viviente. Enfermo, cansado, herido y apenas sin fuerza, andaba con dificultad y no era posible utilizarlo para obtener provisiones de otros lugares. Cuatro días dentro del juego pasaron antes de que todos murieran. El primero falleció debido a las heridas recibidas tras ser atacados por otro grupo durante un asalto 
a nuestro refugio, quienes además se llevaron las pocas provisiones de las que disponíamos. La segunda persona en morir fue la que todavía podía controlar, debido a la enfermedad que arrastraba. Por lo tanto, me quedé con un último personaje, alguien que no se podía mover, con quien no podía hacer nada. Dejé pasar el tiempo sin hacer uso siquiera del botón que permite saltarse el día antes de que termine. Al final, este personaje se suicidó, ahorcándose, y el juego terminó.

Esto es un ejemplo extremo en el que es posible observar cómo los videojuegos, entendidos como experiencias, también liman la agencia del jugador, hasta el punto de sustraerle toda capacidad de acción. En este sentido, también encontré un número importante de entrevistados -y relatos online- que describieron cómo jugar a videojuegos puede tener como resultado experiencias de fracaso y pérdida de agencia:

De repente te salía algo mal, tienes que adaptarte a eso, tú no puedes controlar todo. Entonces tienes que tomar ciertas decisiones que se adapten a esos cambios (Laura, mujer, 26, desarrolladora, artista).

Esto muestra cómo otros agentes poseen la capacidad de condicionar la agencia del jugador, quien, en algunos casos, siente que está perdiendo su habilidad para controlar la situación. Helen Thornham, que llevó a cabo un estudio etnográfico sobre jugadores en varios hogares del Reino Unido, afirma de modo similar que, si los videojuegos tienen el poder de causar interrupciones e interferir en las conversaciones y partidas de los jugadores, entonces debería "ser reconocida su agencia efectiva" (2011: 82). Hay tantos actores que puede llegar a condicionar lo que el jugador está haciendo que resulta muy difícil no tener en consideración su influencia.

Por lo tanto, esta pérdida de agencia que los jugadores sufren con más frecuencia de lo que generalmente es admitido, lleva a los jugadores a experimentar sentimientos de frustración y fracaso. Incluso hay entrevistados que vinculan directamente estas emociones negativas con el mismo acto de jugar a videojuegos: "Frustración es la principal emoción que yo asocio con los videojuegos" (Elisabeth, mujer, 25, jugadora ocasional). Cuando la agencia de los jugadores es interrumpida 0 no ofrece las expectativas esperadas, entonces, la frustración aparece:

Pero si tú juegas a algo que es difícil de manejar 0 apenas responde como debería, se convierte en algo muy frustrante, porque tú pulsas este botón y no está haciendo lo que tú quieres que haga. Eso puede conducir a frustrarte un montón (Andrew, hombre, 28, fuertemente identificado como jugador).

De este modo, los videojuegos pueden ser retratados como dispositivos que, al mismo tiempo, facilitan y condicionan la agencia del jugador. Nos enfrentamos a una paradoja que otros teóricos han encontrado. Por ejemplo, Giddings considera probado que "se actúa sobre los jugadores de videojuegos tanto cómo ellos actúan", centrándose en el evento de jugar "como uno constituido por la traducción lúdica de la agencia — los circuitos excéntricos del efecto y el afecto- entre componentes humanos y no-humanos" (2009: 151). También hay quienes se han acercado a esta paradoja conceptualizándola en términos de balance (Krzywinska, 2007) 0 incluso como una ilusión: "La ilusión de la interactividad promociona un sentido de la agencia - pero esta agencia ha sido predeterminada 0 prediseñada externamente-" (Charles, 2009: 286). Esta aparente contradicción es resumida a la perfección por Tulloch:

El jugador es al mismo tiempo agente activo y prisionero del sistema, protagonista de acontecimientos y esclavo de la autoridad del juego, autor creativo y autómata sin voluntad. Paradójicamente, la idea de juego puede entenderse como supeditada tanto a la agencia como a la docilidad (Tulloch, 2014: 336).

Sin embargo, Tulloch va más allá de este entuerto teórico y ofrece una solución que intenta circunvalar la telaraña en la que la agencia parece quedar atrapada cuando se trata de jugar a videojuegos. Basándose en perspectiva foucaultiana del poder y la agencia, en la que ambos se (re)producen a través de uno y otro en lugar de oponerse mutuamente, Tulloch propone reconocer que las prácticas y las expectativas de los jugadores son "moldeadas por el videojuego, pero al mismo tiempo, el juego sólo se materializa en el momento en el que es jugado por los jugadores" (2014: 348). Ambos son necesarios — junto con 
otros elementos - para existir; son parte de la misma fuerza productiva.

Aunque esté de acuerdo con Tulloch en lo fundamental, y aquí vuelven a reintroducirse las racionalidades asociadas al liberalismo avanzado, considero que subestima cómo la cultura del videojuego - incluyendo una gran parte sus estudiosos - fomenta una representación del jugado como el principal actor activo en términos de responsabilidad: si la condición de posibilidad de la agencia de los jugadores era la libertad de elegir, entonces, tanto sus logros como fracasos son su responsabilidad. Así, incluso habiendo encontrado numerosos entrevistados que reconocen que hay muchos momentos en los que está a merced del videojuego u otros factores, hay una tendencia a revertir los efectos de esta pérdida de agencia, reintroduciendo la importancia del jugador como agente que es capaz de superar estos obstáculos, aunque continúe fallando:

Los jugadores son muy vulnerables, ya sabes, un solo toque y están muertos. Es como si no tuvieran realmente poder porque son muy frágiles. Cuando tú, como jugador, te vuelves más habilidoso, entiendes que tienes todo el poder, ya que tú controlas esos enemigos y puedes matarlos (Noel, hombre, 24, desarrollador, diseñador y programador).

Para este desarrollador que está hablando de su videojuego, una obra reconocida como sumamente difícil, la pérdida de agencia, la fragilidad, y el sentido de falta de poder son temporales. El jugador tiene la habilidad de triunfar, y si falla, será por su falta de destreza o porque no ha invertido suficiente tiempo perfeccionando sus habilidades de juego en esa obra en particular. Como Juul ha aseverado en su ensayo sobre fracaso videolúdico, los videojuegos "nos prometen una oportunidad justa de redimirnos a nosotros mismos", lo que "diferencia el fracaso en el videojuego del fracaso en nuestra vida cotidiana" (2013: 7). Sin embargo, incluso si los videojuegos son más explícitos en lo que tiene que ver con las oportunidades de redención que ofrecen, vemos que hay más similitudes entre el fracaso en los videojuegos y en nuestras vidas cotidianas: en el liberalismo avanzado, los individuos, 0 como mucho grupos 0 comunidades particulares, son señalados como responsables de su propia situación, sin atender a ningún tipo de condicionamiento estructural que pueda estar afectándoles. A este respecto, la cultura del videojuego va de la mano de esta corriente sociopolítica que atraviesa, de forma hegemónica, nuestras sociedades.

Los videojuegos, por lo tanto, interpelan a los jugadores a tomar el mando de lo que tienen delante de ellos — en un sentido literal la mayoría de veces, pues los jugadores suelen usar un mando-y son generalmente señalados como responsables de sus logros y fracasos. Esto es similar a lo que Shaw (2014: 13-39) encontró en las opciones que algunos videojuegos ofertan en términos de representación. En principio, los videojuegos parecen proponer diferentes alternativas a los jugadores para representarse a sí mismos, puesto que se les da la libertad de elegir entre algunas características, incluyendo, raza, género y sexualidad. Pese a todo, al final, el videojuego está colocando la carga de la representación en los hombros del jugador: "antes que incluir diversidad en los videojuegos con personajes ya definidos, la mayoría de representaciones de grupos marginalizados e identificaciones en los videojuegos se deja en manos de los jugadores" (Shaw, 2014: 35).

¿Pero cómo esta noción de agencia en el contexto del videojuego es trasladada al día a día? A continuación, abordaré el rol de esta agencia en relación con diversos aspectos de la vida cotidiana de los jugadores, centrándome en la idea de alcance social.

\section{EL ALCANCE SOCIAL DE LA AGENCIA EN LA VIDA COTIDIANA DEL JUGADOR DE VIDEOJUEGOS}

Cualquier agencia dada tiene, obviamente, efectos limitados, tanto en el espacio como en el tiempo, y el alcance social de su influencia puede variar enormemente dependiendo de diferentes factores. A este respecto, los videojuegos pueden estirar el alcance social de su agencia mediante la extensión de su influencia y, entre otros, facilitar así formas colectivas de agencia y de transferencia de habilidades que fueron aprendidas durante sus sesiones de juego a situaciones de la vida cotidiana. 
Así, los videojuegos abren la posibilidad de articular agencias colectivas, una modalidad de agencia extendida que es fundamental para la construcción de lo que autores como Jenkins (2006) Ilaman cultura participativa. Esta cultura participativa puede ser entendida como la cultura que "absorbe y responde a la explosión de las nuevas tecnologías de la información y comunicación que habilitan al consumidor medio archivar, anotar apropiarse y recircular contenido mediático de poderosas nuevas formas" (Jenkins et al., 2005: 8). La idea principal de la cultura participativa gira en torno a la posibilidad de que cualquiera pueda hacer contribuciones significativas a todo tipo de expresiones culturales.

¿Cómo se traslada esta cultura participativa en el campo de los videojuegos? Guías, mods (modificaciones del software de un juego, que incluye, entre otros, desde cambios y mejoras gráficas a la creación de historias y misiones completamente nuevas), arte y ficciones hechas por fans, creaciones derivadas, intertextualidad, nuevos juegos, sitios web, anuncios, reseñas, interpretaciones, wikis, cosplays (vestirse como personajes de un videojuego, aunque también es aplicable al mundo del cómic, series de televisión o cine), y una lista considerable de acciones que reconocen que "jugar es mucho más que simplemente la interacción de uno 0 unos cuantos individuos con una máquina de videojuegos" (Crawford, 2012: 120). Los jugadores, al usar estas co-creaciones, interpretando esta información y usándola como desean, son parte de una cultura participativa de esa comunidad particular, ya que su experiencia con el juego es mediada por este actor colectivo y, en consecuencia, eso ya ha cambiado tanto al videojuego como a los jugadores.

Los videojuegos fomentan así las interacciones sociales y tienen el potencial de dirigir la agencia hacia formas de colaboración colectiva que, incluso aunque pueda reproducir ciertos aspectos celebratorios del pensamiento liberal y del individuo libre (o comunidad en este caso) que debe cuidar de sí mismo, representa una forma de extender la agencia de los jugadores (y del resto de actores involucrados, humanos o no). Un claro ejemplo de esto se encuentra en el modo en el que videojuegos como Pokémon Go (Niantic Inc., 2016) —que mezclan lo online y lo offline, lo real y lo virtualson capaces de movilizar millones de personas y reconfigurar la relación que se establece entre las personas, la tecnología y el espacio, articulando, de este modo, nuevas formas de agencia compartida y colectiva. En este caso, el videojuego multiplica las interacciones online y offline, dando lugar a un tipo de agencia que se extiende a través de teléfonos móviles, espacios presenciales, entornos virtuales e individuos diversos.

Otro aspecto de la realidad en la que los videojuegos destacan cuando se trata de extender la agencia y su alcance social es la transferibilidad de habilidades adquiridas gracias al juego. Esto prueba que jugar a videojuegos no se encuentra separado de la realidad social y puede tener efectos en otros aspectos de nuestra vida cotidiana más allá de la pantalla:

Creo que los juegos tienen habilidades que son transferibles (...). Pueden llegar a ser útiles en nuestra vida cotidiana, dependiendo de lo que estés haciendo. Como resolver problemas (Zelda, mujer, 25 , se identifica como jugadora).

La agencia es la fuerza que transforma la realidad, pero ésta debe ser encarnada e interpretada, y en esa interpretación la agencia también se transforma. En este caso, después de jugar a videojuegos, las habilidades aprendidas durante ese proceso pueden ser trasladadas a otras situaciones. Los videojuegos median la agencia y la propulsan a través del tejido social. Las fronteras entre el acto de jugar y el contexto social en el que tiene lugar son más porosas de lo que algunos académicos de los estudios sobre videojuegos les gustaría admitir, lo que previene cualquier intento de construir un círculo mágico ${ }^{30}$ (Salen y Zimmerman, 2004:

30 Katie Salen y Eric Zimmerman definen el círculo mágico como "una forma de describir la idea de un espaciotiempo especial creado por el juego. (...) Como círculo cerrado, el espacio que circunscribe está encerrado y separado del mundo real. Como marcador del tiempo, el círculo mágico es como un reloj: representa simultáneamente una senda con principio y final, y una sin origen ni término. El círculo mágico delimita un espacio que se repite, un espacio al mismo tiempo limitado y sin límite. En resumen, un espacio finito con infinitas posibilidades" (2004: 95). 
93-100) que segregue ambos y contenga la agencia únicamente dentro de una interacción directa entre jugador y máquina.

Sin embargo, existen algunas características de los videojuegos que pueden limitar la agencia. En lugar de extenderla, los videojuegos podrían reducirla. El círculo mágico se convierte casi en algo literal y provoca la suspensión temporal de las condiciones sociomateriales de existencia. Las personas están tan inmersas en la experiencia de juego que pierden la noción del tiempo y sólo se preocupan por lo que está ocurriendo en la pantaIla, ignorando todo lo demás.

Supongo que esa es una de las cosas malas de jugar a World of Warcraft o Final Fantasy XI, te puede absorber tanto que puedes olvidar tus otras responsabilidades (Andrew, hombre, 28, fuertemente identificado como jugador).

En este caso, la agencia se concentra casi exclusivamente en lo que está pasando entre el jugador y el videojuego, eludiendo lo que les rodea, y podría llegar tan lejos como descuidar otras cosas importantes en la vida de los jugadores. Cuando la acción de jugar un videojuego funciona como un agujero negro, la agencia queda confinada dentro de los límites de esa interacción y evita que se extienda más allá y alcance cotas sociales más amplias. En perspectiva, este estado de agencia disminuida parece ser menos frecuente entre los entrevistados, pero aun así es uno de los efectos limitantes sobre la agencia a tener en cuenta. Después de todo, la agencia sigue un patrón de extensión e intensión, habilitación y condicionamiento. Su alcance social se modula en función de las mediaciones que provoca y atraviesa.

\section{CONCLUSIONES}

En este texto he explorado la cultura del videojuego con la intención de entender la noción de agencia en la sociedad contemporánea. La obsesión del medio por ofrecer una experiencia de libre albedrío, decisiones difíciles, acciones con consecuencias y un discurso generalizado sobre la centralidad del jugador, me condujeron a plantear que estudiar los videojuegos y su cultura constituiría un campo óptimo en el que acercarse a estas cuestiones relacionadas con la agencia, la libertad, y el poder. Un grupo de conclusiones a nivel ontológico, político y social, que emergen de este proceso, pueden ser dibujadas.

La rica variedad de actores en la cultura de videojuegos sugiere cuestionar la noción tradicional de agencia: el actor social ya no puede ser equiparado de forma exclusiva con la agencia humana; hoy día, estamos gestionando marcos de interpretación de la agencia más complejos que antes. Los videojuegos contribuyen a subvertir el estatus ontológico de la agencia, e indican cómo ha cambiado en las últimas décadas y cómo necesitamos nuevas formas de acercarnos a ella. Siguiendo a Lahti (2003), Millington asegura que los videojuegos son paradigmáticos de la hibridación y de la transgresión de las categorías de lo humano y lo no-humano, lo que ha desdibujado las distinciones entre las personas y las máquinas y demanda una "reconsideración del estatus ontológico concedido a los humanos, creando una necesidad de enfoques epistemológicos novedosos" (2009: 622). La cultura del videojuego es prueba de una noción de agencia ontológicamente promiscua que requiere de nuevas lentes epistemológicas; nuevas lentes que nos ayuden a difuminar la cara genérica de la humanidad como una figura modernista y reconfigurarla en el marco de un paisaje post-humanista (Haraway, 2004: 47) y prostético (Preciado, 2002).

De forma similar, los videojuegos nos muestran que, hoy, la agencia es al mismo tiempo parte de prácticas emancipadoras y alienantes. № sólo la agencia está ligada a la libertad, el empoderamiento y la autonomía, sino que está también asociada a la sumisión, el desempoderamiento y la dependencia. Limitaciones y potencialidades que son parte de los mismos usos de la agencia.

En este sentido, la libertad y la interactividad se encuentran en un contexto que los promociona de un modo que no permite que la agencia rompa los discursos, prácticas y sistemas dominantes. Según Brookey y Booth, en la mayoría de videojuegos "al jugador no se le da la agencia para cambiar las estructuras y diseño de la obra", y por lo tanto se les imponen limitaciones en las opciones que tienen disponibles, lo que "no siempre les facilita el tipo 
de cambios que podrían igualarse con la resistencia ideológica" (2016: 218). Los dispositivos, según la teoría foucaultiana, limitan lo que pasa dentro de ellos, y son el principal ejecutor y manifestación de las condiciones de posibilidad de una sociedad y tiempo específicos (Poltronieri, 2015: 174).

A fin de cuentas, en los videojuegos la agencia está delimitada por el sistema — lo que se puede ver, decir y hacer- como en cualquier otra interacción social mediada por los dispositivos en los que vivimos. Otra cuestión es pensar que el sistema, el dispositivo 0 el aparato tengan contornos bien definidos: los jugadores están limitados 0 habilitados por el videojuego, pero también por los medios de comunicación, los desarrolladores, otros jugadores, y muchos más actores sociales (humanos y no-humanos). Algunos de ellos están cerca en el tiempo y el espacio, otros están actuando a distancia (Latour, 1987: 219-232). En cualquier caso, el lenguaje usado es aquel de la libertad, y de sujetos activos y autónomos capaces de gobernarse a sí mismos; son el lenguaje y práctica del liberalismo avanzado.

Sin embargo, en tanto que estas racionalidades neoliberales fomentan el ideal de sujetos autónomos y autogobernados, están permitiendo que esta agencia produzca líneas de ruptura. En este sentido, el liberalismo avanzado ha sido muy exitoso en dos aspectos: dando opciones a los individuos y haciéndoles responsables de las decisiones tomadas. Se puede decir que las fuerzas del liberalismo avanzado han sido tan exitosas que han plantado la semilla de su declive parcial. Sus racionalidades han promocionado tan fervientemente la libertad individual y la autorresponsabilidad, que han creado un sujeto ampliamente autónomo, uno que, hasta cierto punto, puede ser capaz de escapar de sus propias racionalidades. Después de todo, Deleuze ya consideró que todo dispositivo tiene dos tipos de grupos: "líneas de estratificación o sedimentación y líneas de creatividad" (1990: 165). Las últimas incluyen entre ellas las líneas de "separación, ruptura y fractura" (1990: 162); aquellas que esbozan el movimiento de un dispositivo a otro (1990: 161). Esto apunta a la posibilidad de romper el sistema, como cuando la gente busca glitches en los videojuegos, formas de burlarlo. Los ensamblados de videojuegos (Taylor, 2009) contienen poderosas agencias que podrían llegar a transformar, y de algún modo lo están haciendo actualmente, la realidad.

Podría ser relevante traer a colación la noción de ontopolítica como pensamiento final. La ontopolítica asume que lo real —las condiciones de posibilidad con las que vivimos - no es algo dado, se encuentra "al mismo tiempo abierta y contestada" (Mol, 1999: 75). Esto significa que deberíamos ser capaces de elegir entre diferentes versiones de la realidad 0 , al menos, alentar nuestras preferencias políticas para llevar a cabo "un tipo de realidad antes que otra" (Law, 2004: 13). Tenemos ante nosotros una noción de agencia vinculada al liberalismo avanzado, pero esto podría moverse igualmente hacia la reproducción de sus racionalidades, reforzando el pensamiento y las prácticas liberales, 0 hacia su ruptura, explorando nuevos modos de articular las relaciones de poder e imaginando una agencia que ayuda a transformar la realidad de otras formas, tales como fórmulas más comunitarias de conducirnos a nosotros mismos, compartiendo responsabilidades, reconociendo a aquellos que se encuentran sistemáticamente en una posición de subalternidad, o promocionando la diversidad y la práctica de ponernos en el lugar de otros. Los videojuegos pueden estar sujetos a regímenes ontopolíticos también. Dependerá de su cultura —incluyendo al mundo académico- qué tipo de realidad nos gustaría desarrollar y cuál dejar a un lado.

\section{BIBLIOGRAFÍA}

Arsenault, D., Perron, B. (2009). In the Frame of the Magic Circle: The Circle(s) of Gameplay. En Wolf, Mark J. and Perron, Bernard (editors). The Video Game Theory Reader 2. New York: Routledge, 109-131.

Brookey, R. A. and Booth, P. (2006). Restricted Play. Synergy and the Limits of Interactivity in the Lord of the Rings: The Return of the King Video Game. Games and Culture, 1 (3), 214-230.

Calleja, G. (2011). In-Game: From Immersion to Incorporation. Cambridge, MA: MIT. 
Charles, A. (2009). Playing with one's self: notions of subjectivity and agency in digital games. Eludamos, 3 (2): 291-284. [http://www.eludamos.org/index.php/eludamos/article/view/vol3no2-10/140], acceso 15 de marzo de 2017.

Consalvo, M. (2007). Cheating. Gaining Advantage in Videogames. Cambridge, MA: MIT Press.

Crawford, G. (2012). Video Gamers. London: Routledge.

De Marinis, P. (2005). 16 comentarios sobre la(s) sociología(s) y la(s) comunidad(es). Papeles del CEIC, 15, 1-39, [http://www.ehu.eus/ojs/index. php/papelesCEIC/article/view/12103/11025], acceso 11 de octubre de 2016.

Debord, G. (1995). The Society of the Spectacle. New York: Zone Books.

Deleuze, G. (1990). What is a dispositif? En Armstrong, Timothy J. (editor). Michel Foucault Philosopher. New York: Routledge, 159-168.

Egenfeldt-Nielsen, S., Smith, P. H., Pajares Tosca, S. (2008). Understanding Video Games: The Essential Introduction. New York: Routledge.

Foucault, M. (2003). The Subject and Power. En Rabinow, Paul and Rose, Nikolas. The Essential Foucault. New York: The New Press, 126-144.

García Selgas, F. J. (2007). Sobre la fluidez social. Elementos para una cartografía. Madrid: CIS.

Giddings, S. (2009). Events and Collusions. A Glossary for the Microethnography of Video Game Play. Games and Culture, 4 (2), 144-157.

Gordon, C. (1991). Governmental Rationality: An Introduction. En Burchell, G., Gordon, C., Miller, P. The Foucault Effect. Studies in Governmentality. Chicago: Chicago University Press, 1-51.

Haraway, D. (2004). The Haraway Reader. New York: Routledge.

Jenkins, H. (2006). Fans, Bloggers, and Gamers.

Exploring Participatory Culture. New York: New York University Press.

Jenkins, H., Purushotma, R., Clinton, K., Weigel, M., Robison, A. J. (2005). Confronting the Challenges of Participatory Culture: Media Education for the 21 ${ }^{\text {st }}$ Century. Chicago: MacArthur Foundation.

Juul, J. (2010). A Casual Revolution: Reinventing Video Games and Their Players. Cambridge, MA: MIT Press.

Juul, J. (2013). The Art ofFailure. Cambridge, MA: MIT.
Karppi, T., Sotamaa, 0. (2012). Rethinking Playing Research: DJ HERO and Methodological Observations in the Mix, Simulation \& Gaming, 43 (3), 413-429.

Kirby,A.(2009).Digimodernism.NewYork:Continuum. Krzywinska, T. (2007). Being a determined agent in (the) World of Warcraft: text/play/identity. En Atkins, B., Krzywinska, T. (editors). Videogame, Player, Text. Manchester: Manchester University Press, 101-119.

Lahti, M. (2003). "As We Become Machines: Corporealized Pleasures in Video games". En Wolf, M., Perron, B. (editors). The Video game Theory Reader. New York: Routledge, pp. 157-70.

Latour, B. (1987). Science in Action. Cambridge, MA: Harvard University Press.

Latour, B. (1999a). Pandora's Hope. Essays on the Reality of Science Studies. Cambridge, Mass.: Harvard University Press.

Latour, B. (1999b). On recalling ANT. In Law, J., Hassard, J. Actor-Network Theory and After. Oxford: Blackwell, 15-25.

Latour, B. (2007). Reassembling the Social. An Introduction to Actor-Network-Theory. Oxford: Oxford University Press.

Latour, B. (2008). Reensamblar lo social. Una introducción a la teoría del actor-red. Buenos Aires: Manantial.

Law, J. (2004). After Method. Mess in social science research. London: Routledge.

Lyotard, J.-F. (1984). The Postmodern Condition. Minneapolis: The University of Minnesota Press.

Mäyrä, F. (2012). An introduction to game studies: Games and culture. Thousand Oaks, CA: SAGE.

Miller, P., Rose, N. (2008). Governing the Present. Administering Economic, Social and Personal Life. Cambridge: Polity Press.

Millington, B. (2009). Wii has never been modern: 'active' video games and the 'conduct of conduct'. New Media Society, 11 (4), 621-640.

Mol, A. (1999). Ontological politics. A word and some questions. In Law, J., Hassard, J. ActorNetwork Theory and After. Oxford: Blackwell.

Muriel, D. (2016). Para una sociología de las mediaciones: cartografía impresionista y algunas (breves). Revista Española de Investigaciones Sociológicas, 153, 111-126. 
Preciado, B. (2002). Manifiesto contrasexual. Madrid: Opera Prima.

Poltronieri, F. (2015). Communicology, Apparatus, and Post-history: Vilém Flusser's Concepts Applied to Video games and Gamification. En Fuchs, M., Fizek, S., Ruffino, P., Schrape, N. Rethinking Gamification. Lüneburg: Meson Press.

Rose, N. (1999). Powers of Freedom. Reframing Political Thought. Cambridge: Cambridge University Press.

Salen, K., Zimmerman, E. (2004). Rules of Play: Game Design Fundamentals. Cambridge, MA: MIT Press.

Sayes, E. (2014). Actor-Network Theory and methodology: Just what does it mean to say that nonhumans have agency? Social Studies of Science, 44 (1), 134-149.

Shaw, A. (2014). Gaming at the Edge. Minneapolis: University of Minnesota Press.

Taylor, T. L. (2006). Play between Worlds: Exploring Online Game Culture. Cambridge, Massachusetts: MIT Press.

Taylor, T. L. (2009). "The Assemblage of Play", Games and Culture, vol. 4, 4:331-339.

Thornham, H. (2011). Ethnographies of the Videogame. Gender, Narrative and Praxis. Surrey: Ashgate.
Tulloch, R. (2014). The Construction of Play: Rules, Restrictions, and the Repressive Hypothesis. Games and Culture, 9 (5), 335-350.

Warr, P. (2014). Interview: Warzone Survival In This War Of Mine. Rock Paper Shotgun, [https://www. rockpapershotgun.com/2014/10/25/this-warof-mine-interview/, last accessed 19/04/2016], acceso 18 de noviembre 2016.

\section{NOTA BIOGRÁFICA}

Doctor en Sociología, es investigador en el Instituto de Estudios de 0cio de la Universidad de Deusto. Daniel posee una larga experiencia como investigador y autor sobre cuestiones relacionadas con la identidad, el patrimonio cultural, la juventud, los estudios de ciencia y tecnología, el ocio, el saber experto y los videojuegos. Ha publicado numerosos trabajos en revistas y editoriales de reconocido prestigio como Games and Culture, Ethnic and Racial Studies, Revista Española de Investigaciones Sociológicas, Política y Sociedad, Routledge 0 Anthropos entre otros. Es autor del libro Video Games as Culture (2018). Sitio web: https://danielmuriel.net/. 